\title{
Dynamic changes in leptin distribution in the progression from ovum to blastocyst of the pre-implantation mouse embryo
}

\author{
Laura C Schulz and R Michael Roberts ${ }^{1,2}$ \\ Department of Obstetrics, Gynecology and Women's Health, N625A Health Sciences Center, 1 Hospital Drive, \\ Columbia, Missouri 65212, USA and ${ }^{7}$ Division of Animal Sciences and ${ }^{2}$ Division of Biochemistry, Bond Life Sciences \\ Center, 1201 Rollins Street, University of Missouri, Columbia, Missouri 65211, USA
}

Correspondence should be addressed to L C Schulz; Email: schulzl@missouri.edu

\begin{abstract}
The hormone leptin, which is primarily produced by adipose tissue, is a critical permissive factor for multiple reproductive events in the mouse, including implantation. In the CD1 strain, maternally derived leptin from the oocyte becomes differentially distributed among the blastomeres of pre-implantation embryos to create a polarized pattern, a feature consistent with a model of development in which blastomeres are biased toward a particular fate as early as the two-cell stage. In this study, we have confirmed that embryonic leptin is of maternal origin and re-examined leptin distribution in two distinct strains in which embryos were derived after either normal ovulation or superovulation. A polarized pattern of leptin distribution was found in the majority of both CD1 and CF1 embryos (79.1 and $76.9 \%$ respectively) collected following superovulation but was reduced, particularly in CF1 embryos $(29.8 \% ; P<0.0001)$, after natural ovulation. The difference in leptin asymmetries in the CF1 strain arose between ovulation and the first cleavage division and was not affected by removal of the zona pellucida. The presence or absence of leptin polarization was not linked to differences in the ability of embryos to normally develop to blastocyst. In the early blastocyst, leptin was confined subcortically to trophectoderm, but on blastocoel expansion, it was lost from the cells. Throughout development, leptin co-localized with LRP2, a multi-ligand transport protein, and its patterning resembled that noted for the maternal-effect proteins OOEP, NLRP5, and PADI6, suggesting that it is a component of the subcortical maternal complex with as yet unknown significance in pre-implantation development.

Reproduction (2011) 141 767-777
\end{abstract}

\section{Introduction}

There is considerable evidence that leptin is required for one or more of the developmental processes that allow a conceptus to develop and implant successfully. For example, obese $L e p^{o b / o b}$ mice, which produce only a truncated leptin protein, are infertile, yet fertility may be restored by injecting leptin into $L e p^{o b / o b}$ dams to initiate their reproductive cycles. Moreover, pregnancies can be maintained in such mice by continuing leptin injections through day 6.5 post-coitus (Malik et al. 2001), a result consistent with the prevention of implantation by i.u. injection of a leptin antagonist in wild-type mice (Ramos et al. 2005). Leptin has beneficial in vitro effects on blastocyst formation rates and cell number and the ability to form outgrowths, particularly with respect to trophectoderm, in several species, including cattle (Boelhauve et al. 2005), pig (Kim et al. 2006), and mouse (Kawamura et al. 2003, Herrid et al. 2006, Yang et al. 2006). In one case, human leptin was found to inhibit the development of mouse embryos, but the comparison across species complicates interpretation (Fedorcsak \& Storeng 2003). Treatment with an antibody to leptin receptor (LEPR) neutralized the beneficial effect of leptin on blastocyst development in the mouse, thereby implicating signal transduction through LEPR in mediating leptin action (Kawamura et al. 2002). By contrast, however, Lepr ${ }^{d b}$ mouse conceptuses, which lacked the long form of the receptor, and $\mathrm{Lepr}^{3 /}$ mouse conceptuses, null for all LEPR isoforms, were born at expected ratios to heterozygote mothers (Hummel et al. 1966, Leiter et al. 1980), indicating that there may be no absolute requirement for leptin interaction with its cognate receptors in the embryo for a pregnancy to succeed. This paradox remains unresolved but might imply that an alternative receptor to LEPR is involved in whatever beneficial effects leptin has on the embryonic component of implantation.

In vivo, the embryo is exposed to leptin in both the oviduct and the uterus (Kawamura et al. 2002). In addition to their exposure to exogenous leptin, preimplantation embryos contain leptin, originating from maternal deposition into the oocyte (Cioffi et al. 1997), although there have been conflicting reports as to whether the blastocyst itself expresses Lep mRNA (Kawamura et al. 2003, Herrid et al. 2006), a question 
that we again address in this paper. Controversially, leptin has been observed to be asymmetrically localized in oocytes and zygotes (Antczak \& Van Blerkom 1997) of the CD1 mouse strain. In two-cell embryos, it was confined to the cortical cytoplasm just below the plasma membrane and absent from the zone of cell-cell contact between the two blastomeres. In addition, in some embryos, fluorescence intensity was greater in one blastomere of the two-cell stage embryo than the other. The asymmetry in distribution persisted as blastomeres continued to cleave. At the four-cell stage, there was one blastomere with high leptin content, one with low, and two with intermediate status. From the eight-cell stage onward, leptin was predominantly localized beneath the outer surface plasma membrane of cells on the exterior and absent from inner cells, so that by the blastocyst stage, it was confined to the trophectoderm, with most intense localization to mural trophectoderm. The same authors (Antczak \& Van Blerkom 1999) reported that STAT3, VEGF, BCLX, BAX, TGFB2, VEGF, KIT (C-KIT), and EGFR were also subcortically localized and asymmetrically distributed among early blastomeres.

These data, showing the early embryo to be polarized in terms of protein composition, have been held as evidence that the cytoplasm of the mouse oocyte and zygote is pre-patterned and that the two-cell stage embryo itself is biochemically asymmetrical as a result of differential inheritance of zygotic cytoplasm (Edwards \& Beard 1999). On the other hand, the findings have been controversial (Johnson \& McConnell 2004, Johnson 2009) and, if correct, tend to support the hypothesis that in mammals, as in invertebrates and amphibians, cell lineage determination in the early embryo is influenced by asymmetries in informational macromolecules, a theory that implies that the fate of blastomeres from the two-cell stage onward is somewhat predictable. Others hold a contrary view, namely that all blastomeres are essentially equivalent through the eight-cell stage, at which time location (outer versus inner) and polarization of outside cells become the determinant of differentiation fate. However, both theories recognize that there is flexibility in mammalian embryo development, as separating, moving, or damaging individual blastomeres do not necessarily prevent formation of a viable blastocyst.

Recent evidence suggests that embryos derived via superovulation show a far greater tendency to demonstrate asymmetries in the allocation of cells to trophectoderm and inner cell mass (ICM) than embryos derived by natural ovulation and that there are also differences among mouse strains with regard to these properties (Katayama \& Roberts 2010). It is also known that the properties of the zona pellucida (ZP), especially its shape, might restrict the ability of the embryo to rotate as it advanced toward the blastocyst stage, and that this feature might cause apparent asymmetries without evoking potential fate differences among early blastomeres (Kurotaki et al. 2007, Honda et al. 2008, Katayama \& Roberts 2010). Conceivably, such variables could have contributed to the leptin immunolocalization patterns observed by Antczak \& Van Blerkom (1997). There have been four main goals of this study. The first was to examine whether the polarized pattern of leptin distribution in conceptuses could be confirmed and, if it exists, whether it is dependent on strain and on the ovulation method utilized to generate the zygotes, or on the ZP. The second was to settle the controversy over whether or not Lep transcripts can be detected in mouse conceptuses. The third was to find whether evidence existed for a second mode of leptin mobilization by the conceptus that did not involve the classical cognate receptor LEPR. The final goal was to determine whether there was any link between leptin partitioning into blastomeres and their subsequent developmental fate.

\section{Results}

\section{Immunostaining controls}

Multiple controls were used to ensure the specificity of immunofluorescent staining patterns. When primary antibody was omitted, faint background staining was observed. The background fluorescence from these controls was used to establish image exposure times for the samples co-processed with the controls, such that non-specific background was reduced or eliminated (Supplementary Figure $1 \mathrm{a}$ and $\mathrm{b}$, see section on supplementary data given at the end of this article). Embryos incubated with primary antibody in the presence of 50 -fold (w/w) excess leptin showed only faint background or non-detectable staining (Supplementary Figure 1c). Controls in which detergent was eliminated showed punctate staining on the exterior of the embryo, very different from the diffuse peripheral cytoplasmic staining observed when samples had been permeabilized with detergent (Supplementary Figure 1e). Finally, if the ZP was not removed, the antibody adhered to it non-specifically, and there was a general failure to stain the blastomeres enclosed within it (Supplementary Figure 1f).

\section{Polarization of leptin expression}

Leptin staining was more intense near the periphery of the blastomeres but was absent at cell-cell junctions in the embryos examined from the two-cell stage up to the expanded blastocyst stage (Fig. 1). In addition to this subcortical staining pattern found in all embryos, the leptin expression was classified as polarized when it displayed a similar pattern to that described earlier by Antczak \& Van Blerkom (1997) (Fig. 1III and IV). Specifically, in two-cell embryos, leptin staining was 

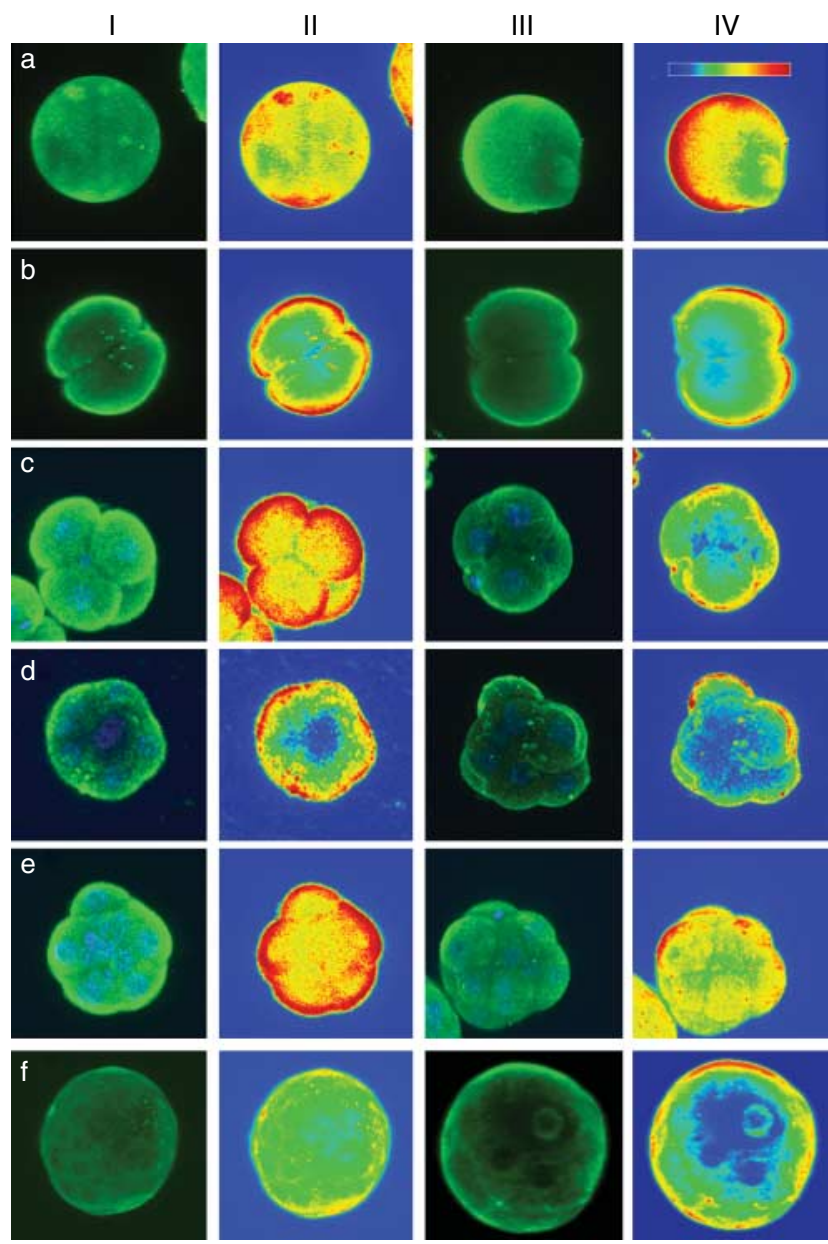

Figure 1 Leptin expression in pre-implantation embryos. Monocolor images of immunofluorescently stained embryos were indicated in pseudocolored green to resemble their appearance on the microscope (columns I and III), or using a heat map scale to visualize differences in staining intensity (columns III and IV), scale bar in column IV a. Representative pictures of ova (a) and embryos examined at the twocell (b), four-cell (c), eight-cell (d), morula (e), and early blastocyst (f) stages. For each embryo examined, the staining pattern was characterized as either 'polarized' (columns III and IV) or 'nonpolarized' (columns I and II). Examples shown are CF1, natural ovulation (columns I-II c- e and columns III-IV d), CF1 superovulation (columns I-II a and III-IV a, C, e), and CD1 superovulation (columns I-II b and $f$, columns III-IV b and f).

considered polarized when it was more intense at one pole of the embryo in both the blastomeres or more rarely, more intense in one blastomere relative to the other (Fig. 1b III and IV). In four-cell embryos, polarized expression was defined as differences in leptin intensity among the four cells, generally with one cell being particularly strongly or weakly stained (Fig. 1c III and IV). At the eight-cell through early blastocyst stages, staining was considered polarized if there was a gradient of intensity across the embryo, with a strongly stained region associated with one pole (Fig. $1 \mathrm{~d}-\mathrm{f} \mathrm{III} \mathrm{and} \mathrm{IV).}$ Conversely, staining was considered to be not polarized if it was of approximately equal intensity at the periphery of all outer blastomeres in the embryo, with no single pole exhibiting markedly increased expression compared with the rest of the circumference (Fig. 1I and II).

Consistent with the findings of Antczak \& Van Blerkom (1997), we found that in both CF1 and CD1 mouse strains almost $80 \%$ of embryos derived from superovulation showed polarized leptin expression (Table 1). On the other hand, these values were reduced when the embryos were obtained after natural ovulation. In the case of the CD1 strain, there was a tendency for fewer embryos from natural ovulation (61.3 vs $80 \% ; P=0.1)$ to show a polarized leptin distribution, whereas in the CF1 strain, the value dropped from $79.1 \%$ following superovulation to $29.8 \%$ following natural ovulation, a change that was highly significant $(P<0.0001)$. It should be noted that we observed a slightly lower prevalence of polarization in CD1 embryos than those observed by Antczak \& Van Blerkom (1997) who reported values $>90 \%$. This discrepancy may be due to minor technical differences. For example, we were unable to detect a gradient when it happened to be oriented precisely in the $z$-axis of the confocal microscope image, because there is a slight loss of signal intensity with increasing depth of field.

Shortly after cavitation, leptin immunofluorescence became limited to the trophectoderm and again was more intense immediately beneath the exterior plasma membrane (Fig. 1f). As at earlier stages, nearly all superovulated $(81.8 \%)$ and the majority of the naturally derived CD1 early blastocysts $(60.0 \%)$ showed polarized leptin expression, whereas in the CF1 strain, polarization was observed in a majority of early blastocysts from superovulated $(60 \%)$ but not in those from naturally ovulated dams, where the value was $37.5 \%$ (Table 1 ). Among those early blastocysts in which polarization was

Table 1 Number of embryos and ova showing polarized leptin expression out of the total number of embryos or ova observed.

\begin{tabular}{llll}
\hline $\begin{array}{l}\text { Mouse } \\
\text { strain }\end{array}$ & Embryo stage & Natural ovulation & Superovulation \\
\hline CF1 & Ovum & 13 of $18(72.2 \%)$ & 12.5 of $18(69.4 \%)$ \\
& Two-cell & 4 of $12(33.3 \%)$ & 10 of $14(71.4 \%)$ \\
& Four-cell & 3 of $14(21.4 \%)$ & 8 of $10(80.0 \%)$ \\
& Eight-cell & 2 of $11(22.2 \%)$ & 11 of $15(73.3 \%)$ \\
& Morula & 2 of $11(22.2 \%)$ & 15 of $16(93.4 \%)$ \\
& Early blastocyst & 3 of $8(37.5 \%)$ & 6 of $10(60.0 \%)$ \\
& Total & 17 of $57(29.82 \%) *$ & 50 of $55(76.9 \%)$ \\
& Two-cell & 4 of $6(66.7 \%)$ & 8 of $9(88.9 \%)$ \\
& Four-cell & 1 of $3(33.3 \%)$ & 7 of $8(87.5 \%)$ \\
& Eight-cell & 7 of $9(77.8 \%)$ & 4 of $6(66.7 \%)$ \\
& Morula & 4 of $8(50.0 \%)$ & 6 of $9(66.7 \%)$ \\
& Early blastocyst & 4 of $6(66.7 \%)$ & 9 of $11(81.8 \%)$ \\
& Total & 19 of $31(61.3 \%)$ & 34 of $43(79.1 \%)$ \\
\hline
\end{tabular}

$* P<0.0001$, the Fisher exact test. 
observed, intense leptin expression was concentrated in the abembryonic pole in seven of nine superovulation-derived CD1 blastocysts, one of three naturally derived CD1 blastocysts, two of three naturally derived CF1 blastocysts, and three of six superovulation-derived CF1 blastocysts.

It was possible to count the number of ICM and trophectoderm cells in 30 of 35 confocal images of these early blastocysts. The relative number of ICM and trophectoderm cells was not different in blastocysts with or without polarized leptin expression, with the ICM comprising $26.9 \%$ of all cells in blastocysts with polarized leptin expression $(n=19)$ and $27.1 \%$ of cells in blastocysts with a non-polarized leptin pattern $(n=11)$. There was no indication of any effect of strain or ovulation method on this percentage.

\section{Leptin expression in expanded blastocysts}

There was a sudden transition in the pattern of leptin immunofluorescence as the blastocyst expanded. In particular, leptin expression was largely lost from the majority of trophectoderm cells. Instead, a region of intense staining, approximating the area of 1-2 cells was observed in $>90 \%$ of all expanded blastocysts examined $(n=22$; Fig. $2 \mathrm{e})$, regardless of strain or ovulation method. This 'spot' was often located close to the junction between trophectoderm, which stained for CDX2 and the unstained ICM. It was not possible to determine with any certainty whether the spot was inside a cell or located at the periphery of the blastocoel cavity, but it in most instances, it appeared to be juxtaposed to 1-2 trophectoderm nuclei (Fig. 2e).

Although it was not possible to follow leptin immunoreactivity in one blastocyst over time, because immunostaining was performed after fixation, some insight into the timing of the phenomenon was gained by fixing groups of CF1 embryos every few hours from the afternoon of day 3.5 until the afternoon of day 4.5. The diffuse cortical leptin staining present in the trophectoderm of all early blastocysts began to fade during the early expansion of the blastocoel cavity, and bright punctate staining became evident (Fig. 2a-d). Multiple larger spots were observed in some embryos in the morning of day 4.5 (Fig. 2b and d), whereas a single very large and intensely labeled spot was observed in more fully expanded blastocysts fixed from cultures in the morning or afternoon of day 4.5 (Fig. 2e and f). This intense spot was mostly, but not entirely, eliminated by incubation with excess leptin (Supplementary Figure 1d), suggesting that it contained either concentrated leptin or leptin remnants. The spot did not appear either when primary antibody was omitted or when other primary antibodies, such as ones against CDX2 (Fig. 2e) were used. The exception to this rule was anti-LRP2 (Fig. 2f, discussed below).
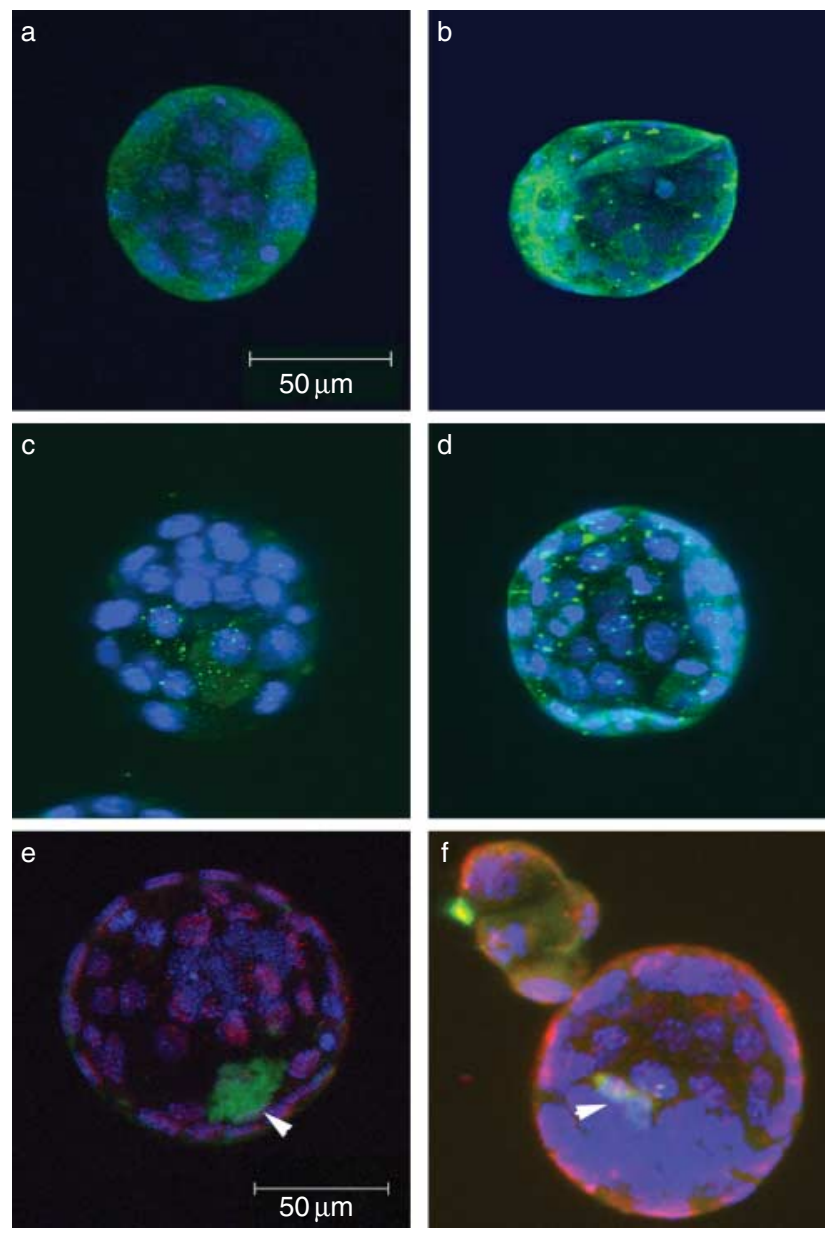

Figure 2 Immunostaining for leptin during blastocyst expansion in CF1 embryos. In two experiments (, , b and C, d), blastocysts were fixed over several hours during blastocyst expansion in vitro. Leptin staining (green) became fainter and punctate (a and c) in the late afternoon of day 3.5 (a) through the early morning (c) of day 4. Larger spots became visible as the morning progressed ( $b$ and d). In fully expanded blastocysts, leptin staining was only visible in a single large 'spot' (arrowhead) that appeared just to the interior of a few trophoblast nuclei (e and f). CDX2 staining was confined to trophectoderm nuclei (red) at this stage (e). Hatching blastocyst stained with both leptin (green) and LRP2 (red) (f). LRP2 was brightest at the periphery of the blastocyst but was also visible in the 'spot' (arrowhead), which appears yellow due to the overlap of green and red staining (f). Scale bar in (a) for images $\mathrm{a}-\mathrm{d}$.

\section{Leptin localization in Swiss Webster embryos in vivo during early pregnancy}

To determine whether the peripheral localization of leptin observed during in vitro embryo culture was an experimental artifact, embryos obtained from natural ovulation were fixed and stained immediately after flushing at the four-cell, eight-cell, and morula stages from Swiss Webster dams (Fig. 3). The leptin staining patterns were similar to those observed in cultured embryos, with leptin predominately located at the 

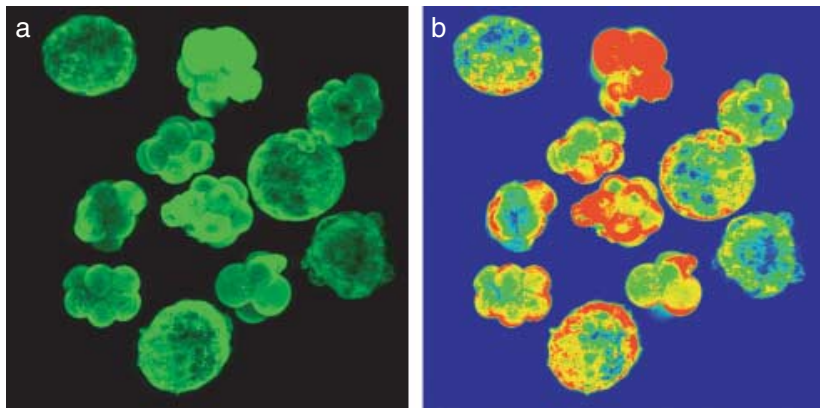

Figure 3 Leptin expression in Swiss Webster embryos from natural ovulation that developed in vivo, without any embryo culture.

The staining pattern is similar to that observed for cultured embryos, with leptin concentrated at the periphery of the embryo, and absent from regions of cell-cell contact. Polarized staining is apparent in some embryos.

periphery of blastomeres, and absent from sites of cell-cell contact and was asymmetrically distributed in some cases.

\section{Origin and maintenance of leptin polarization}

Leptin immunostaining was performed in ova collected from superovulated or naturally ovulated CF1 mice. As in embryos, leptin was predominately localized to the subcortical region of the ova (Fig. 1a, top images). A gradient of leptin expression, with intense staining at one pole and diminished staining intensity at the opposite pole, was also observed in some but not all ova. In contrast to embryos, there was no difference in the prevalence of polarization between naturally and superovulated ova at 70.0 and $66.7 \%$ respectively (Table 1). Thus, polarized leptin expression is apparently lost in naturally ovulated but not superovulated ova between the time when the eggs are ovulated and the first cell division.

\section{Role of the ZP}

To determine whether the ZP is involved in the maintenance of polarized leptin expression and, thus, whether differences in the ZP could account for staining differences between embryos of naturally ovulated and superovulated dams, the ZP was removed from fertilized, superovulated CF1 ova, which were then cultured with ZP-intact controls until day 3.5 when they were at the blastocyst stage. At the four-cell stage, ten of 21 embryos from which the zona had been removed were in either the more common tetrahedral or the less common planar configuration, whereas the remaining 11 had unusual conformations, with all the four blastomeres either in a line or in a $\mathrm{Y}$ or $\mathrm{L}$ shape as observed previously (Katayama \& Roberts 2010). At the morula or early blastocyst stage, the prevalence of polarization in zona intact controls was similar to that observed previously, $73.3 \%(n=15)$, and was only slightly lower, $63.3 \%(n=15)$, in embryos from which the zona had been removed (Fig. 4). These differences were not significant. In other words, the asymmetries observed did not appear to be due to whether a ZP was present.

\section{Embryo leptin production}

To quantify Lep mRNA at the morula and blastocyst stages, real-time RT-PCR was used to construct a standard curve in which 257-2 570000 copies of Lep cDNA could be measured over a linear scale (Supplementary Figure 2, see section on supplementary data given at the end of this article). The data indicate that Lep mRNA, if present at all, was below the limit of detection in small pools of embryos. In each sample, five to eight morulae or blastocysts were pooled in lysis buffer, and total RNA was extracted. Lep mRNA was not detected in any of these pools by RT-PCR (Table 2). Transcripts for a control gene ( $\beta$-actin/Actb) were easily assayed in these embryo RNA pools. Moreover, an adipose tissue sample containing a comparable concentration of Actb mRNA showed Lep expression near the upper end of the standard curve (Table 2; Supplementary Figure 2).
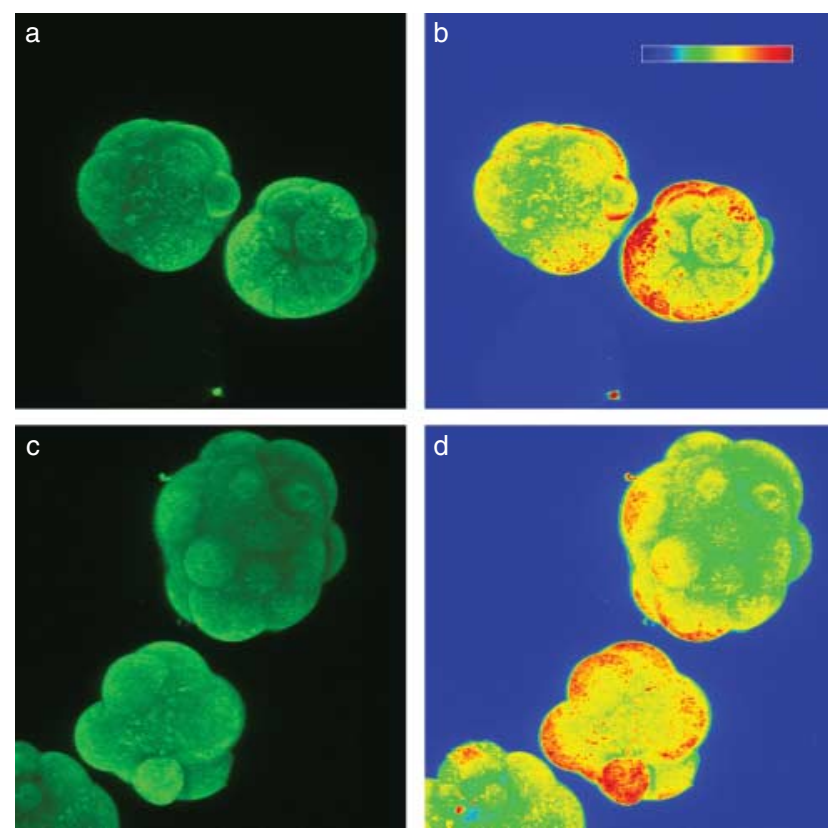

Figure 4 The effect of the removal zona pellucida on leptin polarity. CF1 embryos from superovulation in which the zona pellucida was removed at day 0.5 ( $\mathrm{c}$ and d) and zona intact controls ( $\mathrm{a}$ and b) were cultured through day 3.5 and then fixed. The zona was then removed from the control embryos and all were immunostained for leptin. Monocolor images of immunofluorescence-stained embryos were indicated in pseudocolored green to resemble their appearance on the microscope (a and c) or using a heat map scale to visualize differences in staining intensity (columns b and d). 
Table 2 RT-PCR for leptin and a control gene, $\beta$-actin on RNA obtained by pooling 5-8 embryos.

\begin{tabular}{lll}
\hline Sample & $\boldsymbol{C}_{\mathbf{t}}$ leptin $^{\mathbf{a}}$ & $\boldsymbol{C}_{\mathbf{t}} \mathbf{a c t i n}^{\mathbf{a}}$ \\
\hline No RT control & 35.80 & 37.85 \\
RNA pool 1 - five morulae & Undetectable & 24.14 \\
RNA pool 2 - eight morulae & 35.94 & 22.60 \\
RNA pool 3 - seven blastocysts & Undetectable & 24.37 \\
RNA pool 4 - five blastocysts & 35.93 & 23.25 \\
RNA pool 5 - six blastocysts & Undetectable & 23.64 \\
Adipose tissue RNA & 23.13 & 21.85 \\
\hline
\end{tabular}

$C_{\mathrm{t}}$ is the number of PCR cycles needed to reach fluorescence threshold.

\section{Colocalization with $L D L$ receptor-related protein}

The staining pattern for leptin, with predominant localization to the outer surface of outer blastomeres, resembles the pattern reported for $L D L$ receptor-related protein (LRP2), a protein also known by the names megalin and gp330 (Gueth-Hallonet et al. 1994, Assemat et al. 2005). When CF1 mouse embryos were co-stained for LRP2 and leptin to determine whether the two proteins were co-localized (Fig. 5), both were found in the outer subcortical regions. Areas that stained more intensely for leptin were those that also stained more intensely for LRP2, confirming the likely association of the two antigens within the same region of the peripheral cytoplasm. LRP2 was detectable at all embryonic stages, including the zygote, suggesting that, like leptin, its presence was due to maternal import. At the expanded blastocyst stage, LRP2 staining, unlike that of leptin, was predominately localized near the outer surface of the trophectoderm, as it had been at earlier stages, but it was also associated with the 'spot' of intense leptin staining (Fig. 2f).

\section{Discussion}

The experiments reported in this study largely confirm the leptin distribution data of Antczak \& Van Blerkom (1997) on CD1 mouse pre-implantation embryos. In our confirmatory experiments, we noted two distinct aspects of leptin localization. First, from the two-cell stage onward, leptin was localized to the cytoplasm just beneath the plasma membrane but absent from regions of cell-to-cell contact. By the morula stage, leptin was concentrated in outer and absent from inner cells, and by the early blastocyst stage, it was confined to the trophectoderm. This pattern was independent of strain and ovulation method. Secondly, like Antczak \& Van Blerkom (1997), we noted that the majority of CD1 embryos from the two-cell stage onward demonstrated asymmetries in leptin distribution. It was equally clear, however, that the asymmetries were influenced according to how ovulation was controlled and also by the strain of mouse employed. Embryos from naturally ovulated eggs, particularly of the CF1 strain, frequently showed a fairly uniform distribution of leptin among outer blastomeres, even though the protein had originally been asymmetrically distributed in the ovum (Table 1). This observation, which is novel, has important implications for the developmental significance of leptin asymmetries and adds to the growing body of evidence of significant differences between embryos derived by natural ovulation and superovulation.

Before exploring the distribution of leptin, it was important for us to establish that the leptin observed in our study was of maternal and not embryonic origin. Unlike another report, which used a nested PCR approach (Kawamura et al. 2003), our experiments suggest that the Lep gene is not transcribed from the
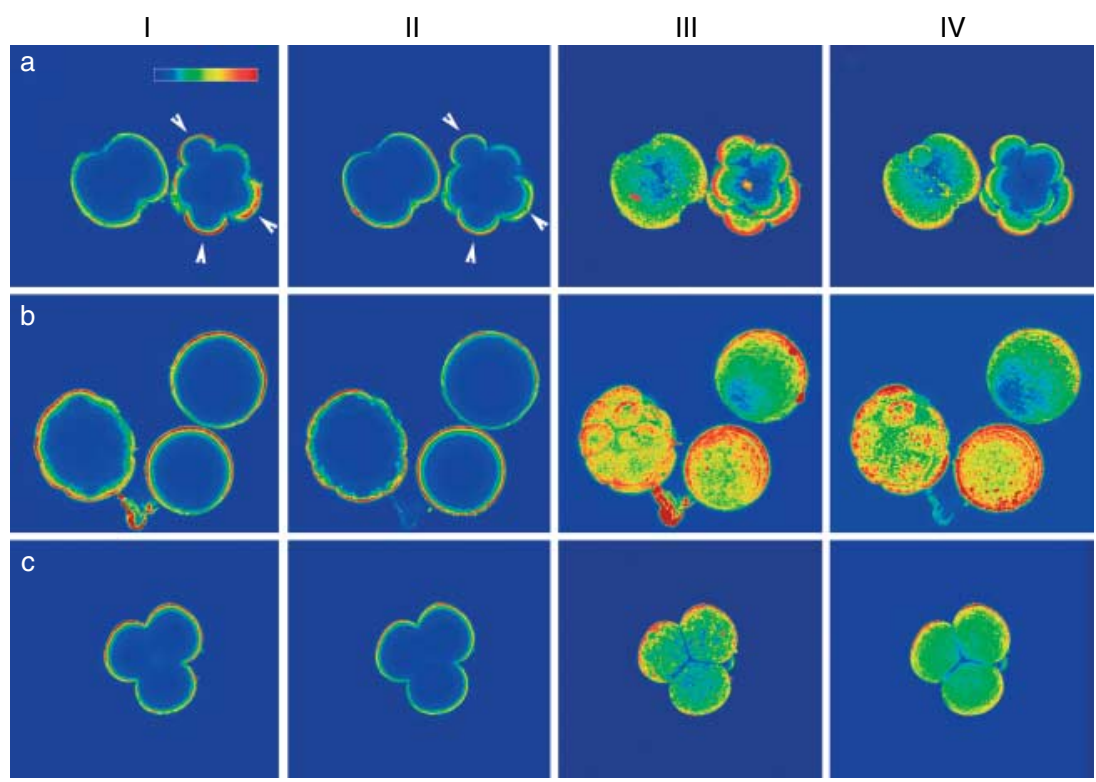

Figure 5 Co-localization of leptin (I and III) and LRP2 (II and IV) by immunostaining of CF1 embryos from superovulation. Single confocal sections (I, II) and three-dimensional reconstructions (III, IV) of a two-cell and eight-cell (a), a morula and two zyogotes (b), and a four-cell (c) are shown. Staining intensity is indicated by the scale bar in (la). The brightness of images was adjusted so that a similar dynamic range is shown for leptin and LRP2 channels. Both leptin and LRP2 staining were more intense at the periphery of embryos, and the regions of more intense staining are similar for leptin and LRP2. For example, in the eight-cell embryo, the same three blastomeres (arrowheads) stained more intensely (red) for LRP2 and leptin. 
embryonic genome through the blastocyst stage of development, in agreement with the results from conventional PCR used by Herrid et al. (2006). Realtime PCR, which allowed detection of as few as 250 copies of Lep mRNA, provided no signal above background from pools of morula or blastocyst cDNA. As there was a minimum of 150 cells ( 5 embryos $\times 32$ cells) contributing to each blastocyst pool, there were $<2$ copies of Lep mRNA per cell. Separate microarray studies performed on two-cell stage blastomeres also indicate that transcripts were below the limits of detection (R M Roberts \& M Katayama, unpublished data). Although we cannot resolve the question of whether there is any embryonic Lep mRNA in mouse embryos, we conclude that the transcript levels present cannot account for the amount of leptin protein observed, thereby supporting the previous contention that all of the leptin present in mouse embryos from the zygote stage to the blastocyst stage is of maternal origin (Antczak \& Van Blerkom 1997, Cioffi et al. 1997).

Maternally supplied leptin must presumably be accumulated through some kind of receptor-mediated uptake into the oocyte and possibly the early embryo (Cioffi et al. 1997). Such transport seems unlikely to depend on the high-affinity, low-capacity cognate receptor, LEPR. In addition, embryos with mutated Lepr genes normally develop (Hummel et al. 1966, Leiter et al. 1980). Conceivably, LRP2, a multi-ligand endocytic receptor associated with clathrin-coated pits and essential for uptake of a wide range of circulating proteins by absorptive epithelia (Fisher \& Howie 2006) and for leptin transport at the blood-brain barrier and in the kidney (Hama et al. 2004, Dietrich et al. 2008), is the responsible transporter in the oocyte and embryo rather than LEPR, which mediates leptin hormonal effects. Consistent with this hypothesis, the most intense regions of leptin staining coincided with equivalently intense regions of LRP2 staining, at least up to the blastocyst stage. In contrast to previous studies (GuethHallonet et al. 1994, Assemat et al. 2005), in which LRP2 was first detected at the eight-cell and 16-cell stages respectively, we observed its presence at all embryonic stages, although LRP2 staining intensity did increase as development progressed.

Some LRP2 even co-localized with leptin to the apparently extracellular 'spots' observed in expanded blastocysts, suggesting that these structures could represent clumps of protein formed after vesicular transport out of the trophectoderm and into the blastocoel cavity. Though this is the first report of leptin being distributed in this manner, similarly located 'spots' have been observed for the protein OOEP (Herr et al. 2008). Moreover, the blastocoel is known to contain other proteins that have been selectively released into the blastocoel cavity (Dardik \& Schultz 1991). It is well established that the endocytic and exocytotic systems of the embryo become progressively more active and polarized as development proceeds and are, of course, essential during blastocyst expansion (Fleming et al. 1986, Pemble \& Kaye 1986). It seems likely that exocytosis of proteins into the blastocoel cavity accompanies bulk transport of fluids, but we can only speculate on why there might be a need to exclude all existing maternal leptin from the trophectoderm at this time. Conceivably, it is important for the embryo to rid itself of once essential proteins to undergo yet another transition, in this case one associated with hatching and implantation.

The subcortical localization pattern we report for leptin has also been observed for LRP2 (Gueth-Hallonet et al. 1994, Assemat et al. 2005), NLRP5 (previously known as MATER; Tong et al. 2000, Ohsugi et al. 2008), OOEP (also called FLOPED and MOEP19; Pierre et al. 2007, Herr et al. 2008, Li et al. 2008, Tashiro et al. 2010), PADI6 (Yurttas et al. 2008), and UCHL1 (Sekiguchi et al. 2006). That this pattern is observed for multiple proteins and is independent of strain and ovulation method suggests functional significance of leptin in the development of the pre-implantation embryo. Some have dubbed this suite of proteins the 'subcortical maternal complex' (SCMC), and others have suggested that it is associated with 'cytoplasmic lattices', filamentous structures unique to the oocyte and early embryo (Capco \& McGaughey 1986, Wright et al. 2003, Li et al. 2008, Kim et al. 2010, Tashiro et al. 2010). Ooep, Uchl1, Nlrp5, and Padi6, in particular, are maternal-effect genes, that is, they are not transcribed by the embryo itself, but embryos from null mothers do not develop beyond the two- to four-cell stage (Yurttas et al. 2008, Li et al. 2010). Unfortunately, it is not possible to test whether leptin is a maternal-effect gene in the same way, as Lep ${ }^{o b / o b}$ dams are anovulatory unless supplied with ectopic leptin.

The reports of asymmetric localization of LEP, STAT3, and a few other proteins within the oocyte, the fourand eight-cell stage embryo, the morula, and the blastocyst in the CD1 mouse (Antczak \& Van Blerkom 1997, 1999) have been received with skepticism (Johnson \& McConnell 2004). However, the fact that the pattern for leptin is reproducible in our hands suggests that the phenomenon deserves some further explanation in terms of more recent models for cell fate decisions in the mouse embryo, such as those described by Piotrowska-Nitsche \& Zernicka-Goetz (2005) and Zernicka-Goetz (2006). This model depicts three cell allocation patterns (Fig. 6). The leptin localization pattern observed in the majority of CD1 embryos, whether obtained by superovulation or by natural ovulation, is consistent with the first pattern of cleavage, i.e. a meridional cleavage occurring first and an equatorial cleavage occurring second (Path 1, illustrated in Fig. 6). The leptin localization patterns observed in CF1 embryos derived from superovulation are consistent with the second cleavage pattern described by Piotrowska-Nitsche 
(1)

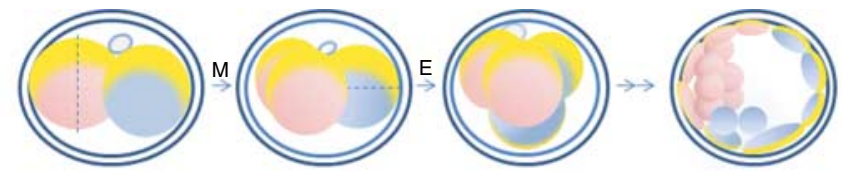

(2)

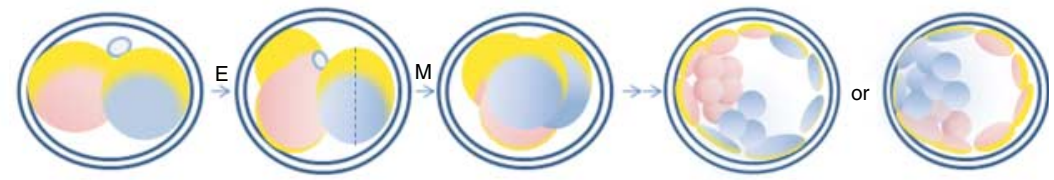

(3)

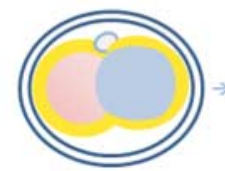

$\overrightarrow{M E}$
$\overrightarrow{E M}$

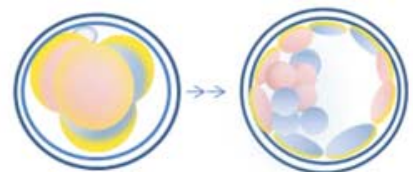

Figure 6 Leptin patterning as evidence for proposed patterns of cell divisions and cell fates in the preimplantation mammalian embryo. Results of leptin staining in CD1 and superovulated CF1 embryos appear consistent with the model of 'biased' development summarized by Zernicka-Goetz. (1) In the biased model, the most common pattern observed from the two-cell stage is a meridional (M) division of the leading (first to divide) blastomere, shown in red, followed by an equatorial division (E), resulting in a tetrahedral shaped four-cell embryo. The lagging blastomere contributes mainly to the embryonic pole of the blastocyst. Leptin (yellow) would be divided equally into daughter blastomeres by an $\mathrm{M}$ division, but would be partitioned unevenly by an E division. Thus, the leptin-rich blastomere would be a product of the lagging cell and would give rise to the abembryonic pole. This is consistent with our observations and those of the Antczak and Van Blerkom in CD1 mice. (2) A less commonly observed pattern in the 'biased' model is an E division followed by an $\mathrm{M}$ division, again resulting in a tetrahedral four-cell embryo with one leptin-rich, one leptin poor, and two intermediate leptin-containing blastomeres. However, when this order of cell divisions was observed by Zernicka-Goetz and others, the leading blastomere was equally likely to contribute to the embryonic and abembryonic pole. Our observations of leptin staining in CF1 embryos from superovulated dams are consistent with this model, with leptin staining observed at either pole. (3) Our observations of leptin staining in CF1 embryos obtained from naturally ovulated dams are not consistent with the 'biased' model. In the majority of these embryos, leptin was absent from sites of cell-cell contact but was otherwise equally distributed along the periphery of all two- and four-cell stage blastomeres, and among all outer blastomeres at later stages.

\& Zernicka-Goetz (2005) and Piotrowska-Nitsche et al. (2005). It predicts that when the first division at the two-cell stage is equatorial and the second division is meridional, the leading blastomere will be equally likely to contribute to the abembryonic or embryonic pole, which is similar to the patterns we observed in CF1 embryos derived by superovulation where the leptin-rich domain in the blastocyst was equally likely to be located at either pole. This observation is also consistent with the previous dye labeling experiments with CF1 embryos derived by superovulation. In these studies, when the progeny of a single blastomere tagged with dye at the two-cell stage was predominantly segregated to one blastocyst pole, it contributed to the embryonic pole $42 \%$ of the time and to the abembryonic pole $58 \%$ of the time (Katayama \& Roberts 2010).

However, the model seems less useful in explaining the leptin distribution in CF1 blastocysts obtained by natural ovulation. In both the dye labeling experiments performed earlier (Katayama \& Roberts 2010) and the leptin staining experiments described in this study, $\sim 70 \%$ of blastocysts from natural ovulation showed a randomized pattern of the marker, with no obvious polarization to one or the other pole. The model of Piotrowska-Nitsche \& Zernicka-Goetz (2005), Piotrowska-Nitsche et al. (2005), and Zernicka-Goetz (2006) predicts that this mixed patterning would occur mainly in embryos that showed a planar configuration at the four-cell stage and generally not in those that had been tetrahedral (Fig. 6, path 3). However, the majority of four-cell stage, zona-enclosed, CF1 embryos derived from natural ovulation retain a tetrahedral configuration (Katayama \& Roberts 2010).

One possible explanation for the different leptin patterns in embryos from natural and superovulation is that the ZP of superovulated ova places more restrictions on planes of cell division and embryo movement within its confines than the ZP of naturally ovulated eggs (Katayama \& Roberts 2010) and is responsible for the predominantly polarized localization of progeny derived from blastomeres tagged with markers at the two-cell stage, which intermix after the eight-cell stage in the absence of the ZP (Motosugi et al. 2005, Kurotaki et al. 2007, Honda et al. 2008). If the properties of the ZP underpinned differences in leptin distribution, the removal of the ZP from CF1 zygotes derived by superovulation would result in loss of leptin polarization. However, this outcome was not observed. One possibility is that leptin patterning is actively maintained and is not solely dependent on the sequence of cleavage divisions. Certainly, the localization of other cortically concentrated proteins such as OOEP and LRP2 are actively regulated, as only in this way could be excluded from sites of cell-cell contact (Gueth-Hallonet 
et al. 1994, Herr et al. 2008, Li et al. 2008). Moreover, the maintenance of leptin polarization in zona-free blastocysts derived from four-cell stage embryos that exhibited the aberrant open chain conformations would also appear to require active relocalization of leptin.

In conclusion, we suggest that the distinctive leptin patterning seen in the CD1 and CF1 mouse strains is primarily due to three factors: first the asymmetric loading of leptin into the oocyte, secondly the sequence and orientation of cleavage divisions in the embryo, and finally active localization of leptin by as yet unidentified binding partners. Superovulation confers a more restrictive pattern of lineage allocation than natural ovulation, particularly in the CF1 strain, but this predictability confers no apparent developmental advantage in terms of increased cell numbers to either ICM or TE (Katayama \& Roberts 2010). Accordingly, there is no evidence to suggest that an asymmetric distribution of leptin (or absence of asymmetry) is linked to abnormal embryonic development. Nevertheless, as maternal leptin is a necessity for normal pregnancy, its essentiality may be linked to its characteristic subcortical location within outwardly facing blastomeres, possibly as a component of the SCMC. Its sudden loss just prior to the time of implantation may reflect the necessary destruction of the complex and its components at that time.

\section{Materials and Methods}

\section{Mouse strains}

CD1 mice are an outbred Swiss-derived, strain, also known as Institute for Cancer Research (ICR), and are derived by Charles River Laboratories (Wilmington, MA, USA) in 1959 from mice donated by the ICR in Philadelphia, which in turn had imported the mice from the Rockefeller Institute in 1926. The CF1 strain was produced by Carworth Farms in the UK from mice obtained via a Missouri Laboratory and thought to have been derived from wild albino mice (Charles River). Accordingly, CD1 and CF1 mice are believed to be genetically distinct. Harlan Swiss Webster mice (ND4; Harlan Laboratories, Indianapolis, IN, USA) originated from the same Rockefeller Swiss strain used to derive CD1 and were later re-derived at the University of Notre Dame (Chia et al. 2005). They are, therefore, likely to be more related to the CD1 strain than to the CF1 strain. All animal experiments conformed to the $\mathrm{NIH}$ Guide for the Care and Use of Laboratory Animals and were approved by the University of Missouri-Columbia Animal Care and Use Committee.

\section{Embryo culture}

The embryos were collected from CD1 (Harlan), CF1 (Harlan and Charles River), and Swiss Webster (Harlan) mice. For superovulation, females were injected intraperitoneally with 5 IU pregnant mare's serum gonadotrophin (Calbiochem, San Diego, CA, USA), followed $46-47 \mathrm{~h}$ later by $5 \mathrm{IU}$ human chorionic gonadotropin (hCG; Sigma, St Louis, MO, USA), at which time they were introduced to male mice of the same strain. Naturally ovulated and superovulated mice were killed for embryo collection at the two-cell stage, $\sim 24 \mathrm{~h}$ after detection of a copulatory plug, i.e. $\sim 36 \mathrm{~h}$ post-coitus. Oviducts were dissected in CZB-Hepes medium on a warming stage, then embryos were transferred to drops of KSOM medium with amino acids (KSOM-aa; Millipore, Billerica, MA, USA) overlain with mineral oil and cultured at $37^{\circ} \mathrm{C}$ under $5 \% \mathrm{CO}_{2}$ and atmospheric oxygen. Ova were collected from the oviducts of CF1 mice following superovulation in the morning after hCG injection, and, following natural ovulation, in the morning after successful mating with a vasectomized male. Cumulus cells were removed with hyaluronidase (Sigma; Katayama \& Roberts 2010).

For comparison of leptin gradients in embryos with and without a ZP, CF1 mice were superovulated as described earlier, and zygotes were collected from the oviduct. Cumulus cells were removed with hyaluronidase and then zygotes washed in CZB-Hepes medium. ZP were removed by exposure to acid Tyrode's solution (Sigma), followed by pipetting in CZB-Hepes solution (Katayama \& Roberts 2010). We cultured one zonaintact embryo and two to four embryos with zona removed in $5 \mu \mathrm{l}$ drops of KSOM-aa medium. All embryos were fixed at day 3.5 post-coitus. All those at the morula or blastocyst stage at the time of fixation were immunostained for leptin.

\section{Immunohistochemistry}

Embryos and ova were fixed in $4 \%$ paraformaldehyde for 15 min immediately after collection (ova and two-cell) or after culture to the four-cell $(\sim 57 \mathrm{~h})$, eight-cell $(\sim 64 \mathrm{~h})$, morula $(\sim 84 \mathrm{~h})$, early $(\sim 89 \mathrm{~h})$ and expanded $(\sim 108 \mathrm{~h})$ blastocyst stages, and then washed and stored in PBS/0.1\% BSA $(\mathrm{w} / \mathrm{v})$ at $4{ }^{\circ} \mathrm{C}$. After fixation, ZP were removed with acid Tyrode's solution (Sigma). The embryos or ova were then incubated for 30-60 min at room temperature in PBS containing 5\% (w/v) IgG-free BSA, and either $5 \%(\mathrm{v} / \mathrm{v})$ normal goat serum or $5 \%$ normal donkey serum $(\mathrm{v} / \mathrm{v})$ to reduce non-specific binding of antibodies in subsequent treatments, and 1\% (v/v) Triton-X-100 to permeabilize cell membranes. Embryos were incubated with antibodies to leptin (catalog \#15-288-20031F chicken antileptin, Genway Biotech, San Diego, CA, USA; now available from Sigma, catalog \# GW20031F), CDX2 (mouse-anti-CDX2, Biogenex, San Ramon, CA, USA), and megalin (LRP2; goat anti-megalin, Santa Cruz Biotechnology, Santa Cruz, CA, USA and rabbit anti-megalin, gift of Joachim Herz) at dilutions of 1:700, 1:100, and 1:100 respectively, overnight at $4{ }^{\circ} \mathrm{C}$ in PBS containing $1 \%$ serum, $1 \%$ BSA, and $0.1 \%$ Triton-X-100. Embryos were washed three times in PBS, $0.1 \%$ BSA. Secondary antibodies (goat anti-chicken Alexa Fluor 488 A11039, goat anti-mouse Alexa Fluor 568 A11031, goat antirabbit Alexa Fluor 568 A11036, and donkey anti-goat Alexa Fluor 568 A11057, Invitrogen; donkey anti-chicken -FITC, Chemicon, Temecula, CA, USA) were used at a 1:500 dilution for $1-2 \mathrm{~h}$ at room temperature. Nuclei were stained with DAPI (Invitrogen). Embryos were washed three times in PBS, $0.1 \%$ BSA, then mounted in a drop of this solution sealed between two coverslips separated by a rubber gasket (catalog \#C18156, Invitrogen). 
Embryos were examined either on a Zeiss LSM 510 twophoton confocal system or a Zeiss LIVE confocal microscope (Carl Zeiss, Thornwood, NY, USA). Embryos only exposed to secondary antibody were used to determine microscope settings so that non-specific signals were subtracted during image acquisitions. Similarly, settings were adjusted so that no part of the image was saturated in order to maximize detection of differences in staining intensity. For each confocal microscopy session, settings were maintained for all embryos imaged on a particular microscope. For the analysis of leptin staining gradients, 3D projections of stained embryos were pseudocolored with LSM Image Examiner software (Zeiss) to envisage differences in staining intensity. In total, two independent observers, one blinded to strain and the other to ovulation method, scored embryos as having 'polarized' or 'non-polarized' leptin expression. In the rare cases of disagreement, the results were averaged. The proportion of embryos showing polarization was compared between natural ovulation and superovulation using a Fisher exact test (GraphPad Prism software, La Jolla, CA, USA).

\section{RT-PCR}

For RNA isolation, CF1 embryos were collected at the two-cell stage and cultured to the morula or blastocyst stage. They were then placed in 'RNA Later' (Ambion, Austin, TX, USA) at $4{ }^{\circ} \mathrm{C}$ for upto $72 \mathrm{~h}$. Trireagent (Sigma) was added and, after phase separation, the aqueous layer was applied to an RNEasy Mini column (Qiagen), and RNA was purified according to the manufacturer's instructions. The RNA was DNase treated and reverse transcribed by using the Quantiscript Kit (Qiagen). To provide a standard, leptin transcripts were PCR amplified from mouse adipose tissue, the band of amplified DNA excised and purified, and DNA concentration measured on a nanodrop spectrophotometer. The PCR product was then serially diluted to create a standard curve. Standard and sample Lep cDNA were amplified using gene-specific primers (F-CAGCCTG CCTTCCCAAAT and R-ATGGAGGAGGTCTCGGAGAT and a FAM-labeled probe (TGCTGCAGATAGCCAATGACCTGG) on an ABI 7500 real-time thermal cycler.

\section{Supplementary data}

This is linked to the online version of the paper at http://dx.doi. org/10.1530/REP-10-0532.

\section{Declaration of interest}

The authors declare that there is no conflict of interest that could be perceived as prejudicing the impartiality of the research reported.

\section{Funding}

This work was supported by NIH Grants K99 HD055231 (to L C Schulz) and R01 HD21896 (to R M Roberts).

\section{Acknowledgments}

The authors wish to thank Mika Katayama for technical assistance and helpful scientific discussion. Jessica Schlitt, Amber Wiggins, and Lisa Mao assisted with analysis of leptin polarity and cell counts.

\section{References}

Antczak M \& Van Blerkom J 1997 Oocyte influences on early development: the regulatory proteins leptin and STAT3 are polarized in mouse and human oocytes and differentially distributed within the cells of the preimplantation stage embryo. Molecular Human Reproduction 3 1067-1086. (doi:10.1093/molehr/3.12.1067)

Antczak M \& Van Blerkom J 1999 Temporal and spatial aspects of fragmentation in early human embryos: possible effects on developmental competence and association with the differential elimination of regulatory proteins from polarized domains. Human Reproduction $\mathbf{1 4}$ 429-447. (doi:10.1093/humrep/14.2.429)

Assemat E, Chatelet F, Chandellier J, Commo F, Cases O, Verroust P \& Kozyraki R 2005 Overlapping expression patterns of the multiligand endocytic receptors cubilin and megalin in the CNS, sensory organs and developing epithelia of the rodent embryo. Gene Expression Patterns 6 69-78. (doi:10.1016/j.modgep.2005.04.014)

Boelhauve M, Sinowatz F, Wolf E \& Paula-Lopes FF 2005 Maturation of bovine oocytes in the presence of leptin improves development and reduces apoptosis of in vitro-produced blastocysts. Biology of Reproduction $\mathbf{7 3}$ 737-744. (doi:10.1095/biolreprod.105.041103)

Capco DG \& McGaughey RW 1986 Cytoskeletal reorganization during early mammalian development: analysis using embedment-free sections. Developmental Biology 115 446-458. (doi:10.1016/0012-1606(86) 90265-4)

Chia R, Achilli F, Festing MF \& Fisher EM 2005 The origins and uses of mouse outbred stocks. Nature Genetics 37 1181-1186. (doi:10.1038/ ng1665)

Cioffi JA, Van Blerkom J, Antczak M, Shafer A, Wittmer S \& Snodgrass HR 1997 The expression of leptin and its receptors in pre-ovulatory human follicles. Molecular Human Reproduction 3 467-472. (doi:10.1093/ molehr/3.6.467)

Dardik A \& Schultz RM 1991 Protein secretion by the mouse blastocyst: differences in the polypeptide composition secreted into the blastocoel and medium. Biology of Reproduction 45 328-333. (doi:10.1095/ biolreprod45.2.328)

Dietrich MO, Spuch C, Antequera D, Rodal I, de Yebenes JG, Molina JA, Bermejo F \& Carro E 2008 Megalin mediates the transport of leptin across the blood-CSF barrier. Neurobiology of Aging 29 902-912. (doi:10.1016/j.neurobiolaging.2007.01.008)

Edwards RG \& Beard HK 1999 Hypothesis: sex determination and germline formation are committed at the pronucleate stage in mammalian embryos. Molecular Human Reproduction 5 595-606. (doi:10.1093/ molehr/5.7.595)

Fedorcsak P \& Storeng R 2003 Effects of leptin and leukemia inhibitory factor on preimplantation development and STAT3 signaling of mouse embryos in vitro. Biology of Reproduction 69 1531-1538. (doi:10.1095/ biolreprod.103.019034)

Fisher CE \& Howie SE 2006 The role of megalin (LRP-2/Gp330) during development. Developmental Biology 296 279-297. (doi:10.1016/ j.ydbio.2006.06.007)

Fleming TP, Cannon PM \& Pickering SJ 1986 The cytoskeleton, endocytosis and cell polarity in the mouse preimplantation embryo. Developmental Biology 113 406-419. (doi:10.1016/0012-1606(86)90175-2)

Gueth-Hallonet C, Santa-Maria A, Verroust P \& Maro B 1994 Gp330 is specifically expressed in outer cells during epithelial differentiation in the preimplantation mouse embryo. Development 120 3289-3299.

Hama H, Saito A, Takeda T, Tanuma A, Xie Y, Sato K, Kazama JJ \& Gejyo F 2004 Evidence indicating that renal tubular metabolism of leptin is mediated by megalin but not by the leptin receptors. Endocrinology 145 3935-3940. (doi:10.1210/en.2004-0074)

Herr JC, Chertihin O, Digilio L, Jha KN, Vemuganti S \& Flickinger CJ 2008 Distribution of RNA binding protein MOEP19 in the oocyte cortex and 
early embryo indicates pre-patterning related to blastomere polarity and trophectoderm specification. Developmental Biology 314 300-316. (doi:10.1016/j.ydbio.2007.11.027)

Herrid M, Nguyen VL, Hinch G \& McFarlane JR 2006 Leptin has concentration and stage-dependent effects on embryonic development in vitro. Reproduction 132 247-256. (doi:10.1530/rep.1.01083)

Honda H, Motosugi N, Nagai T, Tanemura M \& Hiiragi T 2008 Computer simulation of emerging asymmetry in the mouse blastocyst. Development 135 1407-1414. (doi:10.1242/dev.014555)

Hummel KP, Dickie MM \& Coleman DL 1966 Diabetes, a new mutation in the mouse. Science 153 1127-1128. (doi:10.1126/science.153.3740.1127)

Johnson MH 2009 From mouse egg to mouse embryo: polarities, axes, and tissues. Annual Review of Cell and Developmental Biology 25 483-512. (doi:10.1146/annurev.cellbio.042308.113348)

Johnson MH \& McConnell JM 2004 Lineage allocation and cell polarity during mouse embryogenesis. Seminars in Cell \& Developmental Biology 15 583-597. (doi:10.1016/j.semcdb.2004.04.002)

Katayama M \& Roberts RM 2010 The effect of superovulation on the contributions of individual blastomeres from 2-cell stage CF1 mouse embryos to the blastocyst. International Journal of Developmental Biology 54 675-681. (doi:10.1387/ijdb.092942mk)

Kawamura K, Sato N, Fukuda J, Kodama H, Kumagai J, Tanikawa H, Nakamura A \& Tanaka T 2002 Leptin promotes the development of mouse preimplantation embryos in vitro. Endocrinology 143 1922-1931. (doi:10.1210/en.143.5.1922)

Kawamura K, Sato N, Fukuda J, Kodama H, Kumagai J, Tanikawa H, Murata M \& Tanaka T 2003 The role of leptin during the development of mouse preimplantation embryos. Molecular and Cellular Endocrinology 202 185-189. (doi:10.1016/S0303-7207(03)00081-9)

Kim HS, Lee GS, Kim JH, Kang SK, Lee BC \& Hwang WS 2006 Expression of leptin ligand and receptor and effect of exogenous leptin supplement on in vitro development of porcine embryos. Theriogenology 65 831-844. (doi:10.1016/j.theriogenology.2005.06.016)

Kim B, Kan R, Anguish L, Nelson LM \& Coonrod SA 2010 Potential role for MATER in cytoplasmic lattice formation in murine oocytes. PLOS ONE 5 e12587. (doi:10.1371/journal.pone.0012587)

Kurotaki Y, Hatta K, Nakao K, Nabeshima Y \& Fujimori T 2007 Blastocyst axis is specified independently of early cell lineage but aligns with the ZP shape. Science 316 719-723. (doi:10.1126/science.1138591)

Leiter EH, Coleman DL, Eisenstein AB \& Strack I 1980 A new mutation (db3J) at the diabetes locus in strain 129// mice. I. Physiological and histological characterization. Diabetologia 19 58-65. (doi:10.1007/BF00258313)

Li L, Baibakov B \& Dean J 2008 A subcortical maternal complex essential for preimplantation mouse embryogenesis. Developmental Cell $\mathbf{1 5}$ 416-425. (doi:10.1016/j.devcel.2008.07.010)

Li L, Zheng P \& Dean J 2010 Maternal control of early mouse development. Development 137 859-870. (doi:10.1242/dev.039487)

Malik NM, Carter ND, Murray JF, Scaramuzzi RJ, Wilson CA \& Stock MJ 2001 Leptin requirement for conception, implantation, and gestation in the mouse. Endocrinology 142 5198-5202. (doi:10.1210/en.142.12. 5198)

Motosugi N, Bauer T, Polanski Z, Solter D \& Hiiragi T 2005 Polarity of the mouse embryo is established at blastocyst and is not prepatterned. Genes and Development 19 1081-1092. (doi:10.1101/gad.1304805)

Ohsugi M, Zheng P, Baibakov B, Li L \& Dean J 2008 Maternally derived FILIA-MATER complex localizes asymmetrically in cleavage-stage mouse embryos. Development 135 259-269. (doi:10.1242/dev.011445)
Pemble LB \& Kaye PL 1986 Whole protein uptake and metabolism by mouse blastocysts. Journal of Reproduction and Fertility 78 149-157. (doi:10.1530/jrf.0.0780149)

Pierre A, Gautier M, Callebaut I, Bontoux M, Jeanpierre E, Pontarotti P \& Monget P 2007 Atypical structure and phylogenomic evolution of the new eutherian oocyte- and embryo-expressed KHDC1/DPPA5/ECAT1/OOEP gene family. Genomics 90 583-594. (doi:10.1016/j.ygeno. 2007.06.003)

Piotrowska-Nitsche K \& Zernicka-Goetz M 2005 Spatial arrangement of individual 4-cell stage blastomeres and the order in which they are generated correlate with blastocyst pattern in the mouse embryo. Mechanisms of Development 122 487-500. (doi:10.1016/j.mod.2004. 11.014)

Piotrowska-Nitsche K, Perea-Gomez A, Haraguchi S \& Zernicka-Goetz M 2005 Four-cell stage mouse blastomeres have different developmental properties. Development 132 479-490. (doi:10.1242/dev.01602)

Ramos MP, Rueda BR, Leavis PC \& Gonzalez RR 2005 Leptin serves as an upstream activator of an obligatory signaling cascade in the embryoimplantation process. Endocrinology 146 694-701. (doi:10.1210/en. 2004-1186)

Sekiguchi S, Kwon J, Yoshida E, Hamasaki H, Ichinose S, Hideshima M, Kuraoka M, Takahashi A, Ishii Y, Kyuwa S et al. 2006 Localization of ubiquitin C-terminal hydrolase L1 in mouse ova and its function in the plasma membrane to block polyspermy. American Journal of Pathology 169 1722-1729. (doi:10.2353/ajpath.2006.060301)

Tashiro F, Kanai-Azuma M, Miyazaki S, Kato M, Tanaka T, Toyoda S, Yamato E, Kawakami H, Miyazaki T \& Miyazaki J 2010 Maternal-effect gene Ces5/Ooep/Moep19/Floped is essential for oocyte cytoplasmic lattice formation and embryonic development at the maternal-zygotic stage transition. Genes to Cells 15 813-828. (doi:10.1111/j.1365-2443. 2010.01420.x)

Tong ZB, Gold L, Pfeifer KE, Dorward H, Lee E, Bondy CA, Dean J \& Nelson LM 2000 Mater, a maternal effect gene required for early embryonic development in mice. Nature Genetics 26 267-268. (doi:10. 1038/81547)

Wright PW, Bolling LC, Calvert ME, Sarmento OF, Berkeley EV, Shea MC, Hao Z, Jayes FC, Bush LA, Shetty J et al. 2003 ePAD, an oocyte and early embryo-abundant peptidylarginine deiminase-like protein that localizes to egg cytoplasmic sheets. Developmental Biology 256 73-88. (doi:10. 1016/S0012-1606(02)00126-4)

Yang YJ, Cao YJ, Bo SM, Peng S, Liu WM \& Duan EK 2006 Leptin-directed embryo implantation: leptin regulates adhesion and outgrowth of mouse blastocysts and receptivity of endometrial epithelial cells. Animal Reproduction Science 92 155-167. (doi:10.1016/j.anireprosci.2005. 05.019)

Yurttas P, Vitale AM, Fitzhenry RJ, Cohen-Gould L, Wu W, Gossen JA \& Coonrod SA 2008 Role for PADI6 and the cytoplasmic lattices in ribosomal storage in oocytes and translational control in the early mouse embryo. Development 135 2627-2636. (doi:10.1242/dev.016329)

Zernicka-Goetz M 2006 The first cell-fate decisions in the mouse embryo: destiny is a matter of both chance and choice. Current Opinion in Genetics \& Development 16 406-412. (doi:10.1016/j.gde.2006.06.011)

Received 21 December 2010

First decision 3 February 2011

Accepted 28 March 2011 